\title{
6 Erektile Dysfunktion (ED): Diagnostik und Therapie
}

\author{
Dirk Schultheiss
}

\subsection{Einführung}

Eine erektile Dysfunktion (ED) ist definiert als die anhaltende Unfähigkeit, eine für den Geschlechtsverkehr ausreichende Erektion zu erzielen und zu erhalten. Dabei soll der Zustand mindestens 3 bis 6 Monate bestehen und in mindestens $70 \%$ der Versuche ein Geschlechtsverkehr nicht durchführbar sein.

Die sich mit der ED befassende Grundlagenforschung hat ihren Schwerpunkt im Bereich der peripheren und zentralen Mechanismen, die zu einer Relaxation der glatten Muskulatur im Bereich der Corpora cavernosa führen. Auf Grundlage zunehmender Kenntnisse über die intrazelluläre Signalübertragung im Rahmen der Tonusregulation kavernöser, glatter Muskulatur hat sich die Therapie der ED in den letzten drei Jahrzehnten von der Chirurgie (penile Venenchirurgie, Revaskularisationschirurgie und Implantologie) und der intrakavernösen (Schwellkörperautoinjektionstherapie) oder intraurethralen Applikation vasoaktiver Substanzen hin zur oralen, medikamentösen Therapie entwickelt. Heutzutage ist die gute Wirksamkeit und Verträglichkeit der Phosphodiesterase-5-Inhibitoren (PDE-5-Inhibitoren: Sildenafil, Vardenafil, Tadalafil) erwiesen und ihre vorrangige Anwendung etabliert.

Vor der symptomatischen Behandlung einer ED muss eine mögliche kausal therapierbare und therapiepflichtige Genese (z.B. Diabetes mellitus, Fettstoffwechselstörungen, Hypogonadismus [s. Kap. II.11], psychische Ursachen, Beziehungsstörungen) ausgeschlossen bzw. diagnostiziert und therapiert werden. Ebenso können eine Reihe verschiedener Medikamente (Antihypertensiva, Psychopharmaka, Antihistaminika, Lipidsenker, Analgetika und Medikamente mit endokriner Wirkung) einen negativen Einfluss auf die Erektionsfähigkeit haben. Bei reiner oder überwiegend psychogener Ursache besteht die Möglichkeit einer Sexualtherapie, die oft in Kombination mit einer oralen Pharmakotherapie durchgeführt wird. 
Eine Erektionsstörung ist häufig mit einer allgemeinen endothelialen Dysfunktion verbunden und kann somit nur "die Spitze des Eisberges" einer generalisierten Gefäßerkrankung darstellen.

Insbesondere ist die erektile Dysfunktion als Begleitsymptom oder Vorbote einer koronaren Herzerkrankung zu beachten. Zudem gilt, dass sexuelle Aktivität körperlich anstrengend sein kann und mit einer deutlich erhöhten Herzfrequenz und kardialer Belastung einhergeht. Aus diesen Gründen sollte vor medikamentöser Therapie einer Erektionsstörung ein erhöhtes kardiovaskuläres Risiko, vor allem schwere kardiovaskuläre Erkrankungen wie instabile Angina pectoris oder schwere Herzinsuffizienz (New York Heart Association III oder IV), ausgeschlossen werden (Jackson et al. 2010).

Zur Diagnostik und Therapie der ED liegt derzeit keine aktualisierte AWMF-Leitlinie in deutscher Sprache vor, sodass auf englischsprachige Leitlinien zur sexuellen Dysfunktion des Mannes verwiesen werden muss:

- European Association of Urology (EAU) von 2009 (Hatzimouratidis et al. 2010)

- International Consultation on Sexual Dysfunction in Paris 2009 (Montorsi et al. 2010)

- Standards Committee der International Society for Sexual Medicine (ISSM) von 2006 (Porst u. Buvat 2006)

\subsection{Diagnostik}

\subsubsection{Anamnese}

\section{Eine gute Arzt-Patienten-Beziehung hilft die möglichen Ursachen einer erektilen Dysfunktion aufzudecken, die oft eine Aufarbeitung von sehr persönlichen und peinlichen Fragen erfordert. Aus diesem Grund ist besonders der Hausarzt dafür geeignet, diese Evaluation einzuleiten.}

Eine vollständige medizinische Anamnese wie auch eine spezielle Sexualanamnese sollten idealerweise erhoben werden, um einzuschätzen, ob der erektilen Dysfunktion eine organische, psychogene oder multifaktorielle Genese zugrunde liegt. Dabei sollten sich die einleitenden Fragen auf den Beginn der Symptome, das Auftreten und die Dauer partieller Erektionen und das Fortschreiten der erektilen Dysfunktion konzentrieren.

Die Angabe von nächtlichen oder morgendlichen Erektionen kann nützlich für die Unterscheidung einer organischen von einer psychogenen erektilen Dysfunktion sein. Nächtliche Erektionen treten während der REM-Phase des Schlafs auf und setzen ein weitgehend intaktes neurologisches und zirkulatorisches System voraus. Organische Gründe für eine erektile Dysfunktion sind in der Regel charakterisiert durch eine graduelle und persistierende Veränderung der penilen Rigidität oder der Unfähigkeit eine nächtliche, koitale oder durch Selbststimulation erreichte Erektion aufrechtzuerhalten. Der Patient sollte ebenso befragt werden, ob eine Deviation des Penisschaftes oder Schmerzen während der Erektion oder des Koitus bestehen. 
Es ist wichtig, die Libido anzusprechen, da ein verminderter sexueller Antrieb und eine erektile Dysfunktion manchmal frühe Zeichen einer endokrinen Störung (z.B. erhöhter Prolaktin- oder erniedrigter Testosteronspiegel im Serum) sein können.

Weiterhin ist es sinnvoll, danach zu fragen, ob das Problem partnerabhängig ist; eine erektile Dysfunktion entsteht oft in Verbindung mit neuen oder außerehelichen Sexualkontakten. Eine situativ auftretende erektile Dysfunktion suggeriert im Gegensatz zu einer kontinuierlich bestehenden eine psychogene Genese. Die Ejakulation ist sehr viel seltener betroffen als die Erektion; es sollte aber trotzdem erfragt werden, ob die Ejakulation normal, verfrüht, verzögert oder ausbleibend ist. Relevante Risikofaktoren wie Diabetes mellitus, koronare Herzkrankheit, Fettstoffwechselstörungen, Bluthochdruck, periphere Durchblutungsstörungen, Nikotin- und Alkoholabusus sowie endokrine und neurologische Störungen müssen identifiziert werden. Bezüglich der Voroperationen des Patienten interessieren vor allem Darm-, Harnblasen-, Prostata- und Gefäßeingriffe. Eine vollständige Medikamentenanamnese ist ebenfalls wichtig. Von Bedeutung sind auch soziale Lebenseinschnitte, die sich in einer erektilen Dysfunktion niederschlagen können wie gesundheitliche Sorgen, Tod des Lebenspartners, Ehescheidung, Beziehungsprobleme und finanzielle Nöte.

\subsubsection{Körperliche Untersuchung}

Die körperliche Untersuchung ist das nächste wesentliche Element in der Beurteilung einer erektilen Dysfunktion. Nach den Anzeichen einer arteriellen Hypertonie muss gesucht werden, ebenso wie nach Hinweisen auf thyreoidale, hepatische, hämatologische, kardiovaskuläre oder renale Erkrankungen. Eine Untersuchung des endokrinen und vaskulären Systems, des äußeren Genitales sowie der Prostata sollte sich anschließen. Der Penis wird sorgfältig entlang der Corpora abgetastet, um fibrotische Plaques zu erkennen. Ein vermindertes Hodenvolumen oder ein Verlust der sekundären Geschlechtsmerkmale sind Anhalt für einen Hypogonadismus. Die neurologische Untersuchung beinhaltet eine Beurteilung des Analsphinktertonus, die Auslösung des Bulbocavernosusreflexes und die Suche nach einer peripheren Neuropathie.

\subsubsection{Labor}

Ausgewählte Laboruntersuchungen werden prinzipiell bei allen Patienten empfohlen. Dabei sollte zunächst das Gesamttestosteron in den Morgenstunden bestimmt werden und im Falle einer Erniedrigung eine Kontrolle mit erweiterter Hormonanalyse durchgeführt werden, u.a. zur weiteren Differenzierung eines primären (testikulären) gegenüber einem sekundären (hypothalamisch-hypophysären) Hypogonadismus (s. Kap. II.11). Von vielen Untersuchern wird das Serumprolaktin bestimmt, da eine verminderte Libido mit oder ohne erektile Dysfunktion Erstsymptom eines Mikro- oder Makroprolaktinoms bzw. einer Raumforderung im Bereich der Hypophyse mit entsprechender Hyperprolaktinämie sein kann. 
Blutchemie, Blutbild und Lipidprofil können von Wert sein, soweit sie nicht kürzlich bestimmt wurden, da sie Hinweise auf eine Anämie, Diabetes, Hyperlipidämie oder andere systemische Erkrankung geben können, die mit der erektilen Dysfunktion assoziiert sind.

Die Bestimmung des Serum-PSA-Wertes sollte entsprechend der klinischen Leitlinien für Prostataerkrankungen durchgeführt werden und steht nicht in direktem Zusammenhang mit einer ED.

\subsubsection{Erweiterte Diagnostik}

Weitere diagnostische Schritte sind heute seltener notwendig in der Beurteilung einer erektilen Dysfunktion. Bei ausgewählten Patienten können diese Untersuchungen jedoch einen genaueren Einblick in die zugrunde liegenden Pathomechanismen erbringen und in einzelnen Fällen somit eine Hilfe bei der Wahl bestimmter Therapien sein. Methoden dieser erweiterten Diagnostik sind:

1. Messung der nächtlichen penilen Tumeszenz und Rigidität,

2. Gefäßuntersuchungen (intrakavernöse Pharmakotestung, penile Duplexsonographie, penile Angiographie, dynamische Kavernosometrie mit Kavernosographie),

3. neurologische Tests (Messung des Vibrationsempfindens, somatosensorisch evozierte Potenziale) und

4. psychologische Testverfahren.

Der Nutzen der durch diese Verfahren gewonnenen Informationen muss gegen die Invasivität und Kosten der Untersuchungen abgewogen werden.

\subsection{Therapie}

\subsection{1 „First-line“ Therapieoptionen - Orale Therapien PDE-5-Inhibitoren}

Unter sexueller Stimulation kommt es zu einer Aktivierung des NO/CGMP-Signalübertragungsweges und dadurch zu einem Anstieg der intrazellulären 3',5'-cGMP-Konzentration. Dies führt über eine Verringerung des verfügbaren intrazellulären $\mathrm{Ca}^{2+}$ zu einer Relaxation der glatten Schwellkörpermuskulatur.

Die oral verabreichten PDE-5-Inhibitoren entfalten ihre periphere Wirkung über die Hemmung des Abbaus von cGMP. Desweiteren inhibieren die PDE-5-Inhibitoren den aktiven Transport von cGMP aus der Zelle und die Ausschüttng von präsynaptischem Noradrenalin; auch erhöhen sie das intrazelluläre cAMP, wahrscheinlich über eine indirekte Beeinflussung der PDE-3. Diese zusätzlichen Wirkmechanismen sind synergistisch zur PDE-5-Inhibition und erklären die sehr gute klinische Wirksamkeit.

Die Nebenwirkungen und Kontraindikationen der einzelnen PDE-5-Inhibitoren lassen sich durch die Schlüsselfunktionen der Phosphodiesterasen (Soderling u. Beavo 2000) und die spezifische Isoenzymverteilung in Organen und Geweben erklären. 
Alle drei zum jetzigen Zeitpunkt zugelassenen PDE-5-Inhibitoren weisen ein ähnliches Wirkpotenzial auf, unterscheiden sich aber in ihrer Selektivität gegenüber dem PDE-5-Isoenzym, ihrer Pharmakokinetik und ihrem Nebenwirkungsspektrum.

\section{Sildenafil (Viagra $\left.{ }^{\circledR}\right)$}

Der PDE-5-Inhibitor Sildenafil wurde initial auf der Jahrestagung der American Urological Association im Jahre 1996 präsentiert (Boolell et al. 1996). Die Markteinführung erfolgte 1998 zuerst in den USA, seither hat Sildenafil die Therapie von Erektionsstörungen revolutioniert. Sildenafil ist in einer Dosierung von 25, 50 und $100 \mathrm{mg}$ erhältlich. Der Wirkeintritt liegt zwischen 12 und $37 \mathrm{~min}$ (im Mittel $25 \mathrm{~min}$ ), wird jedoch durch fettreiche Nahrungszufuhr und Alkoholgenuss verzögert. Die maximale Plasmakonzentration wird ca. innerhalb einer Stunde erreicht, die klinische Wirkungsdauer beträgt, bei einer Plasmahalbwertszeit von 4-5 h, bis zu 8-12 h. Bei Gabe von 100 mg Sildenafil kann von einer ca. 90\% Hemmung der PDE-5 ausgegangen werden.

Die Ansprechrate unter sexueller Stimulation (,verbesserte Erektion“ in „Global Assessment Question“) ist abhängig von der Genese der Erektionsstörung und liegt zwischen $43 \%$ und 83\%. Bezüglich des Kriteriums „erfolgreicher Geschlechtsverkehr“ schwanken die Zahlen je nach Genese der ED zwischen 48 und 80\%. Die am häufigsten genannten Nebenwirkungen sind konzentrationsabhängig (Goldstein et al. 1998):

- Kopfschmerzen $(10,8 \%)$

- Gesichtsrötung $(10,9 \%)$

- Dyspepsie $(3,0 \%)$

- Rhinitis $(2,1 \%)$

- Schwindel $(2,9 \%)$

- Farbsehstörungen $(1,1 \%)$

Diese unerwünschten Effekte lassen sich durch die Isoenzymverteilung der Phosphodiesterasen im Körper erklären. So kommt die Phosphodiesterase 5 nicht nur im Corpus cavernosum, sondern auch in Gefäßwänden und im Gastrointestinaltrakt vor (Ückert et al. 2001). Auch hemmt Sildenafil schwach die Phosphodiesterase 6, ein Enzym, das im Rahmen der Farbwahrnehmung eine Rolle spielt.

Aufgrund des synergistischen Effektes von PDE-5-Inhibitoren und Nitratverbindungen und/oder NO-Donatoren kann die gleichzeitige Applikation von PDE-5-Inhibitoren zur Vasodilatation und möglicherweise zu einer Hypotension führen und ist deshalb absolut kontraindiziert. Weitere Kontraindikationen sind unter anderem:

- schwere kardiovaskuläre Vorerkrankungen

- Hypotonie

- Retinitis pigmentosa.

Grundlagenwissenschaftliche Untersuchungen konnten einen Effekt von Sildenafil auf Koronararterien und im Myokardgewebe nachweisen. Jedoch zeigte sich in der Häufigkeit des Auftretens von instabiler Angina pectoris und Myokardinfarkten bei Koronarpatienten kein Unterschied bei Einnahme von Sildenafil gegenüber Placebo. Weiterhin belegen epidemiologische Untersuchungen, dass unter der Einnahme von Sildenafil statistisch keine erhöhte Wahrscheinlichkeit von Angina-pectoris-Anfällen, Herzinfarkten oder Todesfällen im Vergleich zu einer alterskorrelierten Ver- 
gleichsgruppe in der Bevölkerung zu verzeichnen ist (Jackson et al. 2010; Vlachopoulos et al. 2009). Patienten, die an einer kardiovaskulären Erkrankung oder einer eingeschränkten ventrikulären Funktion leiden, sollten besonders vorsichtig bzgl. der Wiederaufnahme von sexueller Aktivität sein, um schwerwiegende kardiale Ereignisse zu vermeiden.

Neben der Erschließung neuer Indikationen (pulmonale Hypertonie, koronare Herzerkrankung, chronische Herzinsuffizienz und arterielle Hypertonie) (Ghofrani et al. 2002), wurde außer der On-demand-Therapie der ED auch eine kontinuierliche, prophylaktische Langzeitmedikation mit Sildenafil nach nerverhaltender radikaler Prostatektomie untersucht (Meulemann u. Mulders 2003). Als pathophysiologische Grundlage für das Auftreten einer ED nach radikaler Prostatektomie wird das Fehlen früher postoperativer, insbesondere nächtlicher Erektionen durch Läsionen des Gefäß-Nerven-Bündels intraoperativ vermutet. Chronische Hypoxie und Neuropraxis könnten so zu einer Atrophie der glatten Muskulatur mit zunehmender Fibrosierung führen und wahrscheinlich auch eine Apoptose der cavernösen, glatten Muskulatur induzieren. Vor diesem Hintergrund wurde immer wieder die regelmäßige Gabe eines PDE-5-Inhibitors nach nerverhaltender radikaler Prostatektomie empfohlen; neueren Studien zufolge kann diese Empfehlung jedoch nicht aufrechterhalten werden.

\section{Vardenafil (Levitra $\left.{ }^{\circledR}\right)$}

Seit 2003 steht Vardenafil zur Verfügung. Vardenafil ist eine Substanz, die in vitro ungefähr zehnfach stärker die Hydrolyse von cGMP durch die Phosphodiesterase 5 inhibiert als Sildenafil ( $\mathrm{IC}_{50} \mathrm{O}=0,7 \mathrm{nM}$ gegenüber $6,6 \mathrm{nM}$ ), jedoch eine geringere Bioverfügbarkeit von $15 \%$ aufweist. Vardenafil ist in Dosierungen von 10 und $20 \mathrm{mg}$ erhältlich. Der klinische Wirkungseintritt wird wie bei Sildenafil schon nach 15-30 min und die maximale Plasmakonzentration nach im Mittel 6o min (30-120 min) erreicht. Somit sind Vardenafil und Sildenafil die am schnellsten anflutendenden PDE5-Inhibitoren. Die klinische Wirkung kann bis zu 12 h betragen. Seit 2011 steht Vardenafil als Schmelztablette zur Verfügung, was den Wirkungseintritt nochmals beschleunigt.

Die Wirksamkeit von Vardenafil wurde in verschiedenen randomisierten, placebokontrollierten Studien bei Patienten mit Erektionsstörungen unterschiedlicher Ätiologie und Ausprägung nachgewiesen.

Bezüglich der Verträglichkeit und Anwendbarkeit von Vardenafil bei kardiovaskulären Risikopatienten ergeben sich prinzipiell gleiche Bedingungen wie bei Sildenafil.

Vardenafil wurde auch von Patienten, die zusätzlich eine antihypertensive Medikation (Diuretika, Betablocker, Calciumantagonisten oder ACE-Hemmer) einnahmen, gut toleriert und war wirksam. Die am häufigsten genannten Nebenwirkungen sind denen von Sildenafil, mit Ausnahme der Farbsehstörung, vergleichbar (Young et al. 2001):

- Kopfschmerzen $(10-21 \%)$

- Hautrötung $(5-13 \%)$

- Dyspepsie $(1-6 \%)$

- Rhinitis $(9-17 \%)$

- Sehstörungen $(0,1-<1 \%)$ 


\section{Tadalafil (Cialis ${ }^{\circledast}$ )}

Ebenfalls im Jahr 2003 zugelassen wurde der PDE-5-Inhibitor Tadalafil. Tadalafil ist in den Dosierungen 10 und 20 mg erhältlich. Tadalafil hat eine höhere Affinität gegenüber PDE 11 als Sildenafil und Vardenafil (Eardley u. Cartledge 2002). Die physiologische Bedeutung der PDE 11a, die vor allem in der Prostata, den Hoden und der Skelettmuskulatur nachweisbar ist, ist zum jetzigen Zeitpunkt nicht geklärt (Yuasa et al. 2000). Veränderungen der Testosteron- bzw. Gonadotropinwerte oder der Ejakulatparameter fanden sich bei täglicher Einnahme von $20 \mathrm{mg}$ Tadalafil über 6 Monate nicht (Hellstrom et al. 2002).

Die maximale Plasmakonzentration wird nach 2 h erreicht. Die klinische Wirksamkeit wird wie bei den anderen PDE-5-Inhibitoren schon vorher, jedoch im Mittel später als bei diesen erreicht. Die Plasmahalbwertzeit beträgt 17,5 h, sodass Ansprechzeiten bis zu $36 \mathrm{~h}$ vorkommen.

Zu den am häufigsten genannten Nebenwirkungen zählen:

- Kopfschmerzen (7-21\%)

- Dyspepsie $(1-17 \%)$

- Rhinitis $(5 \%)$

- Cesichtsrötung $(1-5 \%)$

Im Gegensatz zu Sildenafil und Vardenafil können nach der Einnahme von Tadalafil bei Patienten Myalgien (3-7\%) und Rückenschmerzen (4-9\%) auftreten (Porst 2002). Hierfür könnten die inhibierende Wirkung von Tadalafil auf die PDE 11a oder auch die lange Plasmahalbwertzeit verantwortlich sein. Die Nebenwirkung war bei wiederholter Gabe des Medikamentes rückläufig (Brock et al. 2002).

Zusammenfassend zeichnet sich Tadalafil durch eine lange klinische Wirkungsdauer (bis zu 36 h; „Wochenendtablette“) für die Behandlung einer leichten bis schweren Erektionsstörung aus.

Seit 2008 ist Tadalafil auch als Dauermedikation mit einer täglichen Dosis von $5 \mathrm{mg}$ zugelassen. Dies ermöglicht eine spontane sexuelle Aktivität, die nicht an die vorherige zeitgerechte Einnahme eine On-Demand Medikation gekoppelt ist. Zudem wird angenommen, dass sich bei dieser regelmäßigen Medikation positive Effekte des PDE-5-Inhibitors auf die Miktionsbeschwerden bei benigner Prostatahyperplasie bemerkbar machen (Porst et al. 2009).

\section{Yohimbin (Yocon-Glenwood ${ }^{\circledR}$, Yohimbin-Spiegel ${ }^{\circledR}$ )}

Seit über einem Jahrhundert wird das Alkaloid Yohimbin, 1896 von dem Berliner Chemiker Leopold Spiegel aus dem Rindenextrakt des afrikanischen Corynanthe yohimbe Baumes isoliert, zur oralen Therapie von Erektionsstörungen eingesetzt (Schultheiss et al. 2003). Die proerektile Wirkung der Substanz soll vor allem auf einer kompetitiven Blockade zentraler alpha -Adrenozeptoren beruhen. Yohimbin sollte zunächst in einer Dosierung von 3×5 mg (Anfangsdosis über drei Tage) verordnet werden. Anschließend ist eine Steigerung auf $3 \times 10 \mathrm{mg}$ (Erhaltungsdosis) möglich. Eine Verbesserung der Erektionsstörung tritt häufig erst nach 14 Tagen auf; die Einnahme sollte mindestens über sechs Wochen erfolgen. In einer Metaanalyse publizierter Studien (Ernst u. Pittler 1998) konnte eine klinische Wirksamkeit von Yohimbin gefunden werden. Entscheidend ist die Indikationsstellung zur Yohimbintherapie: Signi- 
fikante Verbesserungen der erektilen Funktion treten vor allem bei Patienten mit überwiegend psychogener ED (Versagens- und/oder Erwartungsängste) auf (Hartmann 1997). Für diese Indikation empfahl die Deutsche Gesellschaft für Urologie in ihren alten Leitlinien einen Therapieversuch mit Yohimbin, in der aktuellen Leitlinie der European Association of Urology wird Yohimbin allerdings nicht mehr empfohlen (Hatzimouratidis et al. 2010). Bei insgesamt günstigem Nebenwirkungsspektrum zählen zu den am häufigsten genannten, sehr vereinzelt auftretenden Nebenwirkungen:

- Unruhe,

- Händezittern,

- Schlafstörungen,

- Tachykardien und

- Blutdruckveränderungen.

Aus diesem Grund soll unter der Therapie mit Yohimbin, insbesondere bei Hypertonikern, eine regelmäßige Kontrolle des Blutdruckes erfolgen.

\section{L-Arginin}

L-Arginin haltige Nahrungsergänzungsmittel (Euviril ${ }^{\circledR}$, Prelox ${ }^{\circledR}$ ) sind in letzter Zeit vermehrt auf den Markt gekommen, da mit ihnen in Placebo-kontrollierten Studien bei Männern mit leichter Erektionsstörung eine Wirksamkeit nachgewiesen werden konnte (Ledda et al. 2010).

\section{Androgentherapie}

Die Indikation für eine Testosteronsubstitutionstherapie zur Behandlung einer ED ergibt sich bei nachgewiesener Störung des Androgenhaushaltes, also im Rahmen einer kausalen Substitutionstherapie eines primären oder sekundären Hypogonadismus (s. Kap. II.11). Dies trifft nur für einen geringen Anteil der ED-Patienten zu und erfordert eine weitergehende, spezifische Diagnostik. Bei entsprechender Indikation ist eine orale (eher ungünstig, da nur geringe Testosteronanstiege erzielt werden; Andriol ${ }^{\circledR}$ ), transdermale $\left(\right.$ Androgel $^{\circledR}$, Testogel $^{\circledR}$ ) oder intramuskuläre (Testoviron ${ }^{\circledR}$, Nebido ${ }^{\circledR}$ ) Testosteronsubstitution möglich.

\subsection{2 „Second line“ Therapieoptionen - Lokale konservative Therapien}

Befragt man Patienten mit ED vor Therapiebeginn, so ist die orale Pharmakotherapie die mit Abstand am häufigsten gewünschte Therapieoption. Hier muss aber bedacht werden, dass die Wirksamkeit der oralen Pharmakotherapie über alle Patientengruppen nur ca. 50 bis 60\% beträgt. Weiterhin wissen wir, dass von den Patienten, bei denen ein PDE-5-Inhibitor Wirksamkeit zeigt, nur ca. 50\% eine zweite Verschreibung verlangen. Demzufolge besteht ein hoher, durch die orale Therapie nicht abgedeckter Gesamt-Patientenanteil, dem alternative Optionen angeboten werden müssen.

Es ist von größter Wichtigkeit, den betroffenen Patienten mit ED vor der Initiierung einer oralen Therapie auf diese Tatsachen hinzuweisen und ihm zu erläutern, dass viele weitere Alternativen zur Verfügung stehen, sollte die orale Therapie nicht ansprechen oder er damit nicht zufrieden sein. 


\section{Intraurethrale Therapie}

\section{Prostaglandin $E_{1}\left(\right.$ MUSE $\left.^{\circledR}\right)$}

Die intraurethrale Applikation von PGE wurde zunächst 1996 in den USA und 1999 in Deutschland unter der Bezeichnung MUSE („Medicated Urethral System for Erection“) zugelassen. Das System ist grundsätzlich einfach und patientenfreundlich in der Handhabung und seiner Darreichungsform. Über einen sterilen Applikator wird die in einer Mikrokapsel (Pellet) enthaltene Wirksubstanz in die Harnröhre eingeführt, gelangt über das die Harnröhre umgebende Corpus spongiosum in die Corpora cavernosa und führt dort zu einer Relaxation der glatten Schwellkörpermuskulatur. Der gleiche Wirkstoff ist auch zur direkten Injektion in die Schwellkörper im Rahmen der Schwellkörperautoinjektionstherapie (s.u.) zugelassen. Prostaglandin $\mathrm{E}_{1}$ als MUSE stand initial in einer Dosierung von 250, 500 und $1.000 \mu \mathrm{g}$ zur Verfügung. $\mathrm{Zu}$ den Wirkungsraten stehen unterschiedliche Daten zur Verfügung: In den ersten Studien wurde ein erektionsinduzierender Effekt bei unselektioniertem Patientenkollektiv bei bis zu 70\% der Anwendungen beobachtet (Padma-Nathan et al. 1997), in späteren Studien bei nichtselektionierten Patienten wurden erheblich geringere Ansprechraten (teilweise nur 20\%) sowie hohe Abbruchraten bis zu 70-80\% gefunden (Mulhall et al. 2001). MUSE ist insbesondere für Patienten mit postoperativer ED, bei denen die orale Pharmakotherapie nicht anspricht, attraktiv. Zu den am häufigsten genannten lokalen Nebenwirkungen zählen:

- penile Schmerzen $(24 \%)$

- urethrale Schmerzen (19\%)

- urethrale Blutung $(5,1 \%)$

- Hypotonus $(3-8 \%)$

- vaginale Irritationen $(3 \%)$

- Synkopen $(0,4 \%)$

Priapismen und Fibrosen sind nicht oder nur selten zu erwarten $(<0,1 \%)$. Kontraindikationen bestehen bei Überempfindlichkeit gegenüber dem Wirkstoff, Penisanomalien (Harnröhrenstriktur, schwere Hypospadie, Penisdeviation), Balanitis oder Urethritis.

\section{Schwellkörperautoinjektionstherapie (SKAT)}

\section{Prostaglandin $E_{1}$ (Caverject $^{\circledR}$, Viridal ${ }^{\circledR}$ ) und Papaverin/Phentolamin (Androskat ${ }^{\circledR}$ )}

Im Jahr 1982 wurde erstmals über die intrakavernöse Injektion von Pharmaka zur Behandlung der Erektionsschwäche berichtet. Die Beschreibung der Injektiontherapie durch Brindley und Virag gilt als ein Meilenstein in der Therapie der ED (Schultheiss et al. 2003). Heute stehen verschiedene Wirkstoffe bzw. Wirkstoffkombinationen zur Verfügung: Als erste Wahl gilt das Alprostadil ( $\mathrm{PGE}_{1}$ ), weiterhin wird auch die Kombination Papaverin/Phentolamin (in Deutschland nicht für diese Indikation zugelassen) angewendet. Die Schwellkörperautoinjektionstherapie wurde durch die Einführung der oral wirksamen Therapiemöglichkeiten weitgehend verdrängt. Jedoch ist die sehr wirksame und bei sachgemäßer Anwendung nebenwirkungsarme Injektionstherapie für Patienten, bei denen eine orale, systemische Therapie kontraindiziert oder unwirksam ist, eine gute Alternative.

Die Wirkmechanismen der einzelnen Substanzen werden wie folgt beschrieben: Papaverin wirkt auf intrazellulärer Ebene als nichtselektiver Phosphodiesteraseinhibi- 
tor. Phentolamin ist ein nichtspezifischer alpha-Adrenozeptorblocker, entfaltet also seine lokale Wirkung nach Injektion in das Schwellkörpergewebe auf Rezeptorebene. Allein verabreicht, bewirkt Phentolamin keine ausreichende Erektion; die Kombination aus Phentolamin und Papaverin stellt aber eine wirkungsvolle Therapieoption der ED dar.

Über die lokale, erektionsauslösende Wirkung von PGE $_{1}$ wurde erstmals 1986 berichtet. Die Bindung erfolgt an spezifischen, zellmembranständigen Rezeptoren und führt über eine komplexe intrazelluläre Regulationskaskade zu einer Relaxierung der Schwellkörpermuskulatur.

Bei der Schwellkörperautoinjektionstherapie sind zunächst eine Austestung der optimalen, individuellen Dosierung sowie eine ausführliche ärztliche Einweisung in die Anwendung unabdingbar. Die Kombination aus Papaverin ( $15 \mathrm{mg} / \mathrm{ml})$ und Phentolamin $(0,5 \mathrm{mg} / \mathrm{ml})$ wird in einer Dosierung von o, $2 \mathrm{ml}$ bis zu $2 \mathrm{ml}$ angewendet, Alprostadil $\left(\mathrm{PCE}_{1}\right)$ steht in einer Dosierung von 5-20 $\mu \mathrm{g}$ zur Verfügung. Die Wirkung der Kombination von Phentolamin und Papaverin ist mit Ansprechraten von 60-90\% in zahlreichen klinischen Studien gut dokumentiert. Gute Ergebnisse werden insbesondere bei Patienten mit neurogener oder psychogener erektiler Dysfunktion erreicht. Die Wirksamkeit des Kombinationspräparates ist etwas geringer als die von Alprostadil $\left(\mathrm{PGE}_{1}\right)$. Das Kombinationspräparat sollte aufgrund der deutlich höheren Rate prolongierter Erektionen nur bei Unverträglichkeit oder Versagen von $\mathrm{PGE}_{1}$ angewandt werden. Zu den weiteren Nebenwirkungen von Phentolamin/Papaverin zählen Schwellkörperfibrosen, Penisverkrümmungen und Schmerzen (1-7\%). Bei Patienten mit Alkoholabusus oder Leberschäden sollte die Indikation zur Therapie mit Phentolamin und Papaverin zurückhaltend gestellt werden.

Das zur Schwellkörperautoinjektion in Deutschland zugelassene Alprostadil ( $\left.\mathrm{PGE}_{1}\right)$ zeigt mit 70 bis über 90\% die höchsten Ansprechraten aller pharmakologischen Therapieformen der ED. Häufigste Nebenwirkungen sind:

n milde bis mäßige, penile Schmerzen im (5-30\%)

- Schwellkörperveränderungen (Fibrose, Penisverkrümmungen, Verhärtungen; 3-8\%)

- prolongierte Erektionen (ca. 2-3\%, unter 1\% der Injektionen)

Ist bei maximaler Dosierung von Phentolamin/Papaverin oder Prostaglandin $\mathrm{E}_{1}$ keine für den Geschlechtsverkehr ausreichende Erektion zu erreichen, ist eine Kombination der drei Wirkstoffe („Triple-Mix“) möglich. Hierbei ergaben sich hohe Ansprechraten von über 90\%. Unter Umständen ist eine Reduzierung der Einzeldosierungen und dadurch der mit den entsprechenden Wirkstoffen verbundenen Nebenwirkungen möglich.

\section{Vakuumerektionshilfen}

Vakuumerektionshilfen spielen bei der heutigen Therapie der ED zwar eine geringere Rolle, sind aber eine fest etablierte und nicht invasive Therapieoption und können eine sinnvolle Alternative für die Patienten sein, welche PDE-5-Inhibitoren nicht einnehmen sollten und/oder andere Maßnahmen ablehnen. In den EAU Leitlinien werden die Vakuumerektionshilfen daher als First line Therapie eingestuft. Sie bewirken durch den erzeugten Unterdruck eine passive Blutfüllung der Schwellkörper, wobei dann ein an der Peniswurzel angebrachter Gummiring einen Blutabfluss und den da- 
mit verbundenen Erektionsabfall verhindert. Nebenwirkungen der sogenannten „Vakuumpumpen“ sind Schmerzen, Taubheitsgefühl, Hauteinblutungen (Marcumar!) und eine gestörte Ejakulation. Zudem beklagen viele Patienten, dass das Gerät unhandlich sei und die erreichte Erektion einen unnatürlichen Charakter habe. Zur Optimierung der Wirksamkeit sollten Patienten, die sich für diese Therapieoption entscheiden, eine individuelle Unterweisung in die sachgerechte Handhabung erhalten.

\subsection{3 „Third line“ Therapieoptionen - Operative Therapien}

Zu den operativen Therapieoptionen der ED zählen neben den penilen Implantaten die Venen- und Revaskularisationschirurgie, wobei die beiden letzteren Verfahren Ausnahmeindikationen darstellen und als experimentell anzusehen sind. Beide Verfahren weisen schlechte Langzeitergebnisse auf und haben allenfalls noch bei der Therapie einer hochselektiven Patientengruppe ihre Berechtigung.

Die penilen Implantate (fälschlich oft auch als „Penisprothesen“ bezeichnet) hingegen zeichnen sich, eine entsprechende Indikationsstellung (schwere ED, Versagen der konservativen Therapie oder entsprechende Kontraindikationen) und Patientenaufklärung vorausgesetzt, durch hohe Akzeptanz und Zufriedenheit bei entsprechender Haltbarkeit und guten Langzeitergebnissen aus. Der Einsatz von Implantaten wird aufgrund der hohen Invasivität als „Third-line-Option“ der ED-Therapie nach den Leitlinien der EAU eingestuft (Hatzimouratidis et al. 2010). Neben den mehrheitlich implantierten, hydraulischen 3-Komponentensystemen (gute Steuerbarkeit und gutes kosmetisches Ergebnis) stehen semirigide Implantate (geringere Kosten und leichte Handhabung) zur Verfügung. Zu den eingriffstypischen Komplikationen zählen die Implantatinfektion, der Funktionsverlust einzelner Komponenten einschließlich Leckage sowie die Perforation von Haut oder Urethra. Die im Rahmen von Multicenterstudien ermittelten 5-Jahresfunktionsraten lagen in Abhängigkeit des verwendeten Implantattyps zwischen 84 und 94\% bei einer Patienten- und Partnerinnenzufriedenheit in über $85 \%$ der Fälle.

\section{Zusammenfassung}

Mit den drei PDE-5-Inhibitoren Sildenafil, Vardenafil und Tadalafil stehen hochwirksame, orale Substanzen als Therapieoption der ersten Wahl zur Verfügung. Die Substanzen weisen ein ähnliches Wirkpotenzial auf, unterscheiden sich aber in ihrer Selektivität gegenüber den PDE-Isoenzymen, ihrer Pharmakokinetik und ihren Nebenwirkungsprofilen. Neben der etablierten On-demand-Therapie der ED kann eine kontinuierliche tägliche Langzeitmedikation mit PDE-5-Inhibitoren in geringer Dosierung vorteilhaft sein. Eine prophylaktische Behandlung nach ein- oder beidseitig nerverhaltender radikaler Prostatektomie wird nach neuesten Untersuchungen eher kritisch gesehen.

Im Bereich der lokalen Pharmakotherapie gilt die intrakavernöse Applikation von Prostaglandin $E_{1}\left(P_{G E}\right)$ als Mittel der Wahl bei Nonrespondern auf PDE-5-Inhibitoren bzw. bei entsprechenden Kontraindikationen für PDE-5-Inhibitoren. „Triple-Mix“ mit PGE ${ }_{1}$, Papaverin und Phentolamin bleibt PGE Nonrespondern vorbehalten. Die transurethrale Applikation von $\mathrm{PGE}_{1}\left(\mathrm{MUSE}^{\circledR}\right)$ ist eine Alternative der lokalen Pharmakotherapie insbesondere für Patienten mit mittelgradiger Erektionsstörung.

Vakuumerektionshilfen können eine sinnvolle Alternative für Patienten sein, bei denen PDE-5-Inhibitoren kontraindiziert sind oder die allgemein medikamentöse Therapien ablehnen. In den EAU Leitlinien werden die Vakuumerektionshilfen daher auch als First line Therapie eingestuft. 
Die operative Versorgung mit semirigiden oder hydraulischen Penisimplantaten findet weiterhin Anwendung bei Versagen aller medikamentösen Therapieformen, bzw. bei Ablehnung der Verfahren oder bestehenden Kontraindikationen. Die penile Gefäßchirurgie, entweder als venöse Widerstandserhöhung oder als arterielle Revaskularisation, ist heute auf sehr wenige und ausgelesene Indikationen reduziert und wird nur noch von wenigen Zentren durchgeführt.

\section{Literatur}

Boolell M, Allen M), Ballard S, Gepi-Attee S, Muirhead GI, Naylor AM, Osterloh IH, Gingell C (1996) Sildenafil: An orally active type 5 cGMP-specific phosphodiesterase inhibitor for the treatment of penile erectile dysfunction. Int I Impot Res 8, 47-52

Brock GB, McMahon CG, Chen KK, Costigan T, Shen W, Watkins V, Anglin G, Whitaker S (2002) Efficacy and safety of tadalafil for the treatment of erectile dysfunction: Results of integrated analyses. I Urol 168, 1332-1336

Eardly I, Cartledge I (2002) Tadalafil (CIALIS) for men with erectile dysfunction. Int I Clin Pract 56, 300-304

Ernst E, Pittler MH (1998) Yohimbine for erectile dysfunction: A systematic review and meta-analysis of randomized clinical trials. J Urol 159, 433-436

Ghofrani HA, Wiedemann R, Rose F, Schermuly RT, Olschewski H, Weissmann N, Gunther A, Walmrath D, Seeger W, Grimminger $F(2002)$ Sildenafil for treatment of lung fibrosis and pulmonary hypertension: a randomized controlled trial. Lancet 360, 895-900

Goldstein I, Lue TF, Padma-Nathan H, Rosen RC, Steers WD, Wicker PA (1998) Oral sildenafil in the treatment of erectile dysfunction. N Engl / Med 338, 1397-1404

Hartmann U (1997) Psychologische Diagnostik und Sexualanamnese. In: Stief CG, Hartmann U, Höfner K, Jonas U (Hrsg.) Erektile Dysfunktion. Springer, Berlin Heidelberg New York, S. 105-115

Hatzimouratidis K, Amar E, Eardley I, Giuliano F, Hatzichristou D, Montorsi F, Vardi Y, Wespes E (2010) Guidelines on male sexual dysfunction: erectile dysfunction and premature ejaculation. Eur Urol 57, 804-814

Hellstrom W|, Overstreet I, Yu A, Shen W, Beasley C, Watkins V (2002) Tadalafil has no effect on semen characteristics. Int I Impot Res 14, 29-30

Jackson G, Boon N, Eardley I, Kirby M, Dean J, Hackett G, Montorsi P, Montorsi F, Vlachopoulos C, Kloner R, Sharlip I, Miner M (2010) Erectile dysfunction and coronary artery disease prediction: evidence-based guidance and consensus. Int I Clin Pract 64, 848-857

Ledda A, Belcaro G, Cesarone MR, Dugall M, Schönlau F (2010) Investigation of a complex plant extract for mild to moderate erectile dysfunction in a randomized, double-blind, placebo-controlled, parallel-arm study. BJU Int 106, 1030-1033

Meulemann EJH, Mulders PFA (2003) Erectile function after radical prostatectomy: A review. Eur Urol 43, 95-102

Montorsi F, Adaikan G, Becher E, Giuliano F, Khoury S, Lue TF, Sharlip I, Althof SE, Andersson KE, Brock G, Broderick G, Burnett A, Buvat I, Dean I, Donatucci C, Eardley I, Fugl-Meyer KS, Goldstein I, Hackett G, Hatzichristou D, Hellstrom W, Incrocci L, Jackson G, Kadioglu A, Levine L, Lewis RW, Maggi M, McCabe M, McMahon CG, Montague D, Montorsi P, Mulhall I, Pfaus |, Porst H, Ralph D, Rosen R, Rowland D, Sadeghi-Nejad H, Shabsigh R, Stief C, Vardi Y, Wallen K, Wasserman M (2010) Summary of the recommendations on sexual dysfunctions in men. I Sex Med 7, 3572-3588

Mulhall JP, Jahoda AE, Ahmed A, Parker M (2001) Analysis of the consistency of intraurethral prostaglandin E(1) (MUSE) during at-home use. Urology 58, 262-266

Padma-Nathan H, Hellstrom WJG, Kaiser FE, Labasky RF, Lue TF, Nolten WE, Norwood PC, Peterson CA, Shabsigh R, Tam PY, Place VA, Gesundheit N (1997) Treatment of men with erectile dysfunction with transurethral alprostadil. Medicated Urethral System for Erection (MUSE) Study Group. N Engl I Med 336, 1-7

Porst H (2002) IC 351 (Tadalafil, Cialis): update on clinical experience. Int I Impot Res 14, 57-64

Porst H, Buvat I (2006) Standard Practice in Sexual Medicine. Blackwell Publishing, Oxford

Porst H, McVary KT, Montorsi F, Sutherland P, Elion-Mboussa A, Wolka AM, Viktrup L (2009) Effects of once-daily tadalafil on erectile function in men with erectile dysfunction and signs and symptoms of benign prostatic hyperplasia. Eur Urol 56, 727-735

Schultheiss D, Stief CG, Jonas U (Hrsg.) (2003) Klassische Schriften zur erektilen Dysfunktion: Eine kommentierte Sammlung von Originaltexten aus drei Jahrtausenden. ABW, Berlin 
Soderling SH, Beavo JA (2000) Regulation of CAMP and cGMP signaling: New phosphodiesterases and new functions. Curr Opin Cell Biol 12, 174-179

Ückert S, Küthe A, Stief CG, Jonas (2001) Phosphodiesterase isoenzymes as pharmacological targets in the treatment of male erectile dysfunction. World J Urol 19, 14-22

Vlachopoulos C, loakeimidis N, Rokkas K, Stefanadis C (2009) Cardiovascular effects of phosphodiesterase type 5 inhibitors. I Sex Med 6, 658-674

Young I, Auerbach S, Porst H (2001) Vardenafil, a new selective PDE inhibitor, significantly improved all IIEF domains and showed favourable safety profile in patients with erectile dysfunction over 12 weeks. J Urol 165, 224

Yuasa K, Kotera I, Fujishige K, Michibata H, Sasaki T, Omori K (2000) Isolation and characterization of two novel phosphodiesterase PDE11 A variants showing unique structure and tisse-specific expression. I Biol Chem 275, 31469-31479 